\title{
Study of Virtual Interaction System based on Micro-mechanical Sensor and Motion Builder
}

\author{
Guolong Zhang ${ }^{1, \text { a }}$, Xinglu Wang ${ }^{2, \mathrm{~b}}$, Yongsheng Wang ${ }^{1, \mathrm{c}}$, Xin Wang ${ }^{1, \mathrm{~d}}$ \\ ${ }^{1}$ School of Art \& Design, Lanzhou Jiaotong University, Lanzhou, Gansu, 730070 \\ ${ }^{2}$ School of Mechanical Engineering, Lanzhou Jiaotong University, Lanzhou, Gansu, 730070 \\ a email, ${ }^{\mathrm{b}}$ email, ${ }^{\mathrm{c}}$ email, ${ }^{\mathrm{d}}$ email
}

Keywords: Motion Capture System, Virtual Interaction, Wireless Sensor Network, Motion Builder

\begin{abstract}
By using the human motion capture system for mechanical sensors, this paper makes the virtual actor is imitated in real time in Motion Builder and the motion of the real performer is imitated accurately. A set of human motion capture virtual interactive system is designed. This system mainly involves the human body model real-time reconstruction software design, the sensor technology, the sensor network node design, the ZigBee wireless communication technology and so on.
\end{abstract}

\section{Introduction}

In the computer hardware technology, inertial sensor technology and applications, animation games and the rapid development of film and television industry today, people in the creation of animation and film and television production of special effects on the increasingly high demand, so the movement capture technology in which To the crucial role and wide range of applications. In addition, various industries also pay special attention to various types of motion capture systems in terms of operational efficiency, real-time performance, and cost reduction. In this paper, through the micro-mechanical sensors, wireless communication technology and three-dimensional animation software Motion Builder application, designed a set of human motion capture virtual interactive system. The system can be real-time, accurate on the screen to reproduce the reality of human motion. In the design of human body motion capture virtual interaction system, it is divided into the overall design of the system and the related design of the software system integrating the existing hardware system.

\section{Design of Virtual Interaction System for Human Motion Capture}

Motion capture technology has developed rapidly in recent years, its variety, through the principle of classification and the mainstream motion capture technology can be divided into the following four categories: acoustic, electromagnetic, mechanical and photoelectric motion capture technology. Based on the actual development environment and the future use environment, this paper adopts the human motion capture system based on the micromechanical sensor developed by Lanzhou Jiaotong University. It is a set of motion capture system based on mechanical motion capture technology Capture system, the system is mainly based on sensor technology and zigbee wireless data communication technology. Mechanical motion capture technology has excellent real-time, capture the relatively high precision, the work environment is almost no restrictions, etc., and has a very high price. In the software design of this system, the aim is to simulate the action of the real human body in real time by the three-dimensional animated character created on the computer, so the main motion data processing software is used: Motion Builder software. Motion Builder software has a wealth of tools and motion simulation production system, the action data processing, the role of action matching and output of the final effect also has a very high quality.

Based on sensor technology and zigbee wireless data communication technology, the human motion capture system based on micromechanical sensor developed by Lanzhou Jiaotong University can capture the human body's motion and attitude data with high accuracy and real-time, 
and has high performance-price ratio. This system is assigned to 17 motion attitude measurement sensor units, in turn distributed in the human body 17 key joints. 17 micro-mechanical sensors are composed of three-axis acceleration sensor, three-axis angular velocity sensor, three-axis magnetic field sensor and MCU highly integrated, with high accuracy and real-time. The sensor network node and the main controller module is connected with the bus type structure, and uses RS485 to carry on the communication. The main function of the sensor network is to obtain the motion data of the joints of the human body in real time and to aggregate and transmit the data. By studying the motion posture of the human body, the motion attitude measurement sensor is placed at the 17 key joints with the greatest influence. Simultaneously, the data nodes are bound in the virtual animated characters in the graphic display module in synchronization with the 17 key joints, and real-time and accurate data of the performers are acquired in real time. Through the real-time movement of the performer data processing, effective in the computer is to imitate the real human posture. In the 17 key nodes in the movement of the human body is divided into left and right itself, respectively, placed two main controller, respectively, handle two half-body key joint movement data, the two sensor nodes are independent of each other, and Each of the sensor cells in each of the two sensor networks has its own unique ID. These two sensor networks form the sensor network module of the whole motion capture system. Real-time and accurate real human and motion attitude data acquired by the sensor network are transmitted to the wireless data receiving module connected with the computer through the JN5139 wireless data transmission module. The JN5139 wireless transmission and receiving module is a wireless communication hardware used in the motion capture system. It has the advantages of high integration, long communication distance and low cost. It is highly integrated with RF components and microcontrollers. It is easy to use and users can develop ZigBee and other protocols of various types of wireless communication systems. In this way, performers can not only get rid of the inconvenience brought by cable, but also can have a greater movement environment, and can get a higher degree of freedom of movement. This module data transmission has distance, but also for the design of this system.

Human motion capture virtual interactive system software is mainly based on motion data processing software Motion Builder to achieve. Motion Builder software mainly by the data transfer station module and graphic display module of the two modules, which is responsible for the action capture system through the designated port to send real-time data collection And simulates real-time motion of real-life performers synchronously with the virtual characters created in the three-dimensional modeling software.

The data transfer station module is part of the human interaction design software. First of all, the human-body motion capture system based on MEMS sensor network to real-time collection of the movement of the human node data sent through the computer's designated port to the module, the use of socket communication technology, the hardware collected by the various sensors The node data is transferred to the graphic display module in real time. Therefore, the data transfer station module is the source of the entire system data.

Graphic display module is mainly through the motion data processing software Motion Builder to develop and implement. In Motion Builder software integrates socket communication client program, it is mainly used for real-time hardware data acquisition of each sensor module. Motion Builder software also provides developers with a powerful SDK that integrates a series of modular function functions, by calling the specified function can be specified by the human joints and virtual role model of the movement of data corresponding to the node binding, thus driving the virtual characters to carry out synchronous movement. 


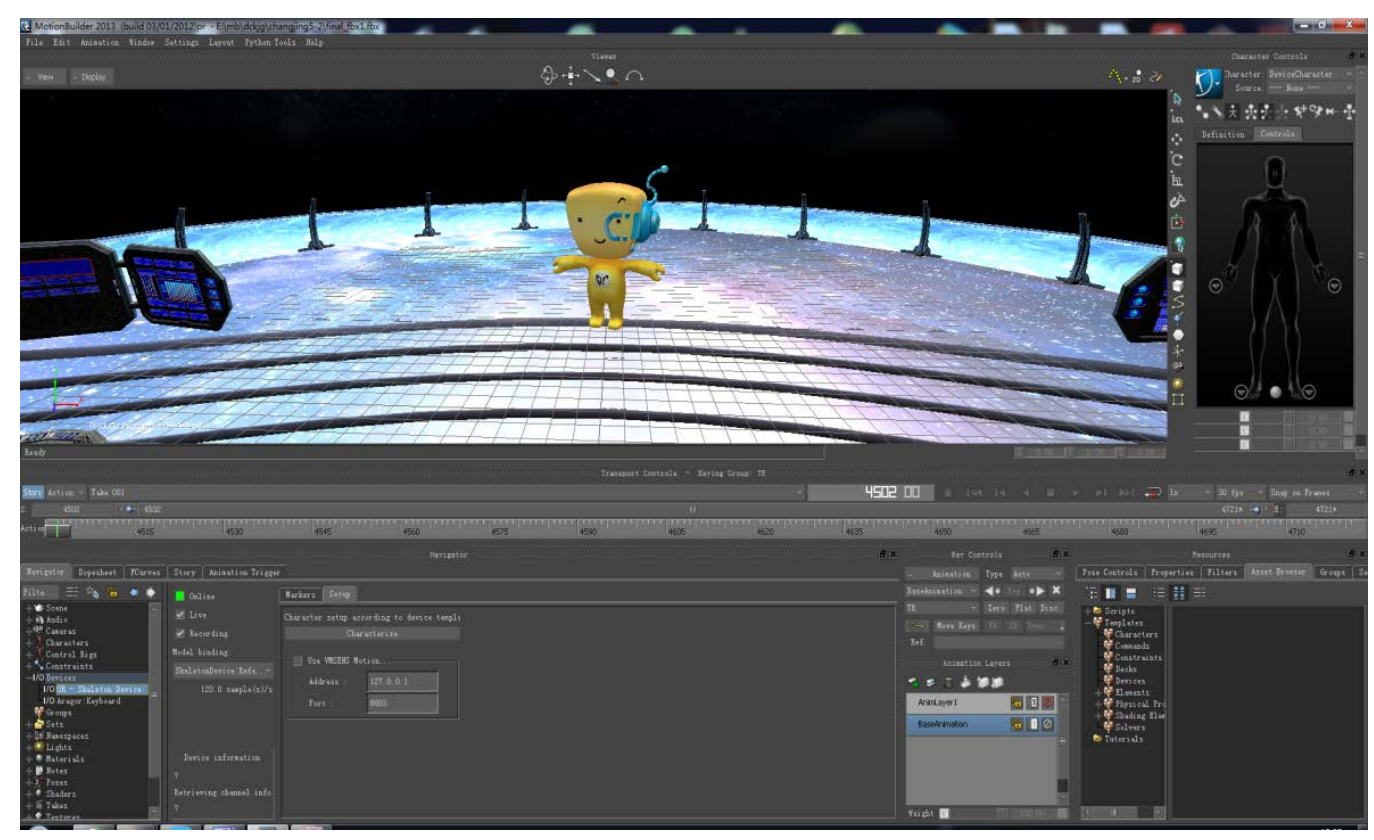

Figure 1 based on Motion Builder human motion capture virtual interactive system

\section{Human Motion Capture Virtual Interactive System Specific Implementation Steps}

In the motion capture system based on the micromechanical sensor, the movement attitude data are collected and distributed in real time by the sensor network distributed in the 17 key joints of the moving human body. The motion data of each node is finally transmitted by the main controller module and the wireless data transmission module To the system workstation, the workstation has a corresponding wireless data receiving module is responsible for real-time data reception, and through the serial communication to the data transfer module to the module. The figure shows the body of the human motion capture system, the human body wearing the figure device, that is, the performer put on a sensor with 17 tights renderings.

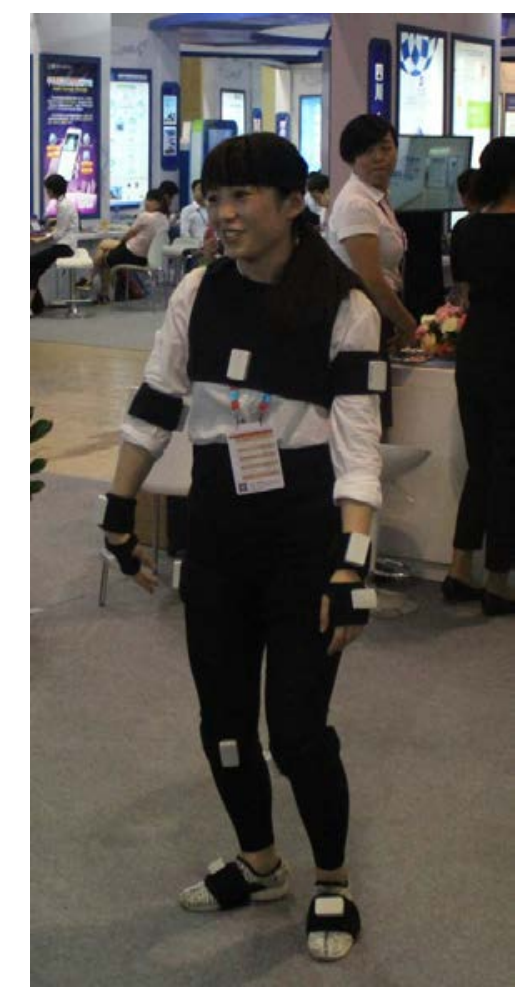

Figure 2 The performer wears a 17-sensor tights view

In the computer and wireless data transmission module link, the need for data transfer module in 
the 17 sensor network to read the movement of data. Need to carry on simple port setting operation in its data transit software. The following figure shows the serial port program interface for data acquisition.

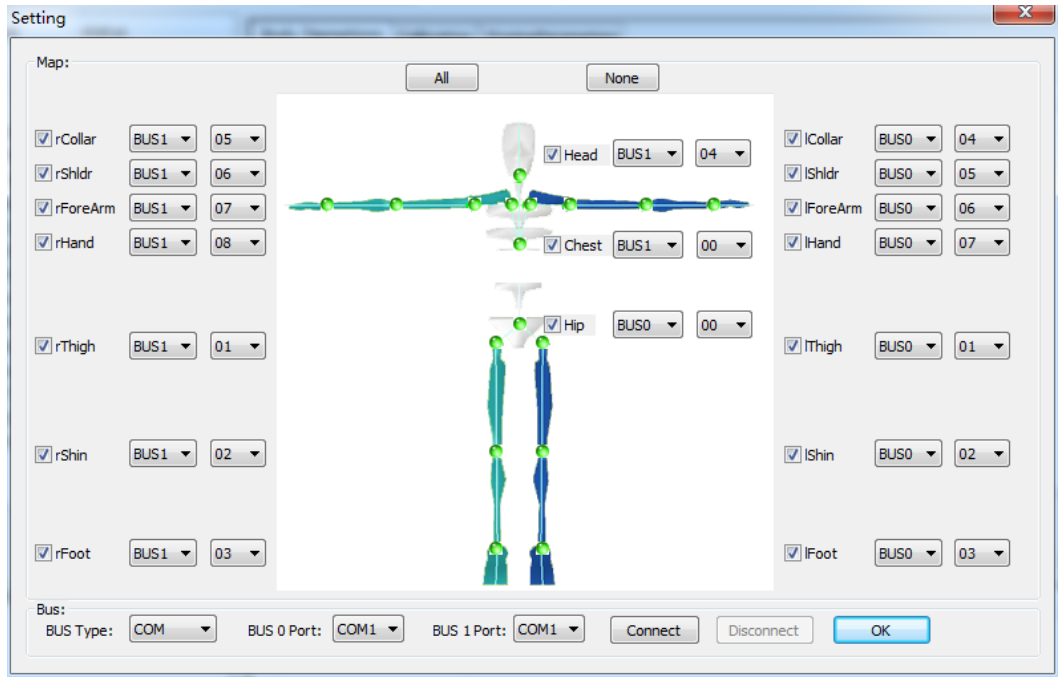

Figure 3 data acquisition serial port program interface settings interface

When the performer has been wearing a good action capture system 17 sensor module, and through the corresponding serial port for data collection and transmission work, the performer's gesture data through the socket communication sent to the graphical display module, In the middle of the graphic display module can be achieved to imitate and reproduce the performer gesture. In the graphic display module, this is accomplished by loading devices, binding data between templates and nodes in Motion Builder software, creating model templates, setting socket communication addresses, and model template characterization operations. In the Motion Builder software to load the virtual animation role and the real body is also distributed on the body of the 17 key joints, through the motion capture system sensor network to capture the real human movement and posture data in the virtual animation role in the body of the 17 key joints One by one correspondence, to achieve the role of virtual animation to imitate and reproduce the gesture of the work of performers.

In Motion Builder software, you first need to start the graphics display module and data transfer module between the data communication function, where the system uses socket-based data communication, graphical display module is the corresponding socket client program. First, set the Address and Port of the motion capture system in I / O Devices, and open the Online switch. Check the use of the Motion Capture system module. The operation details are as follows:

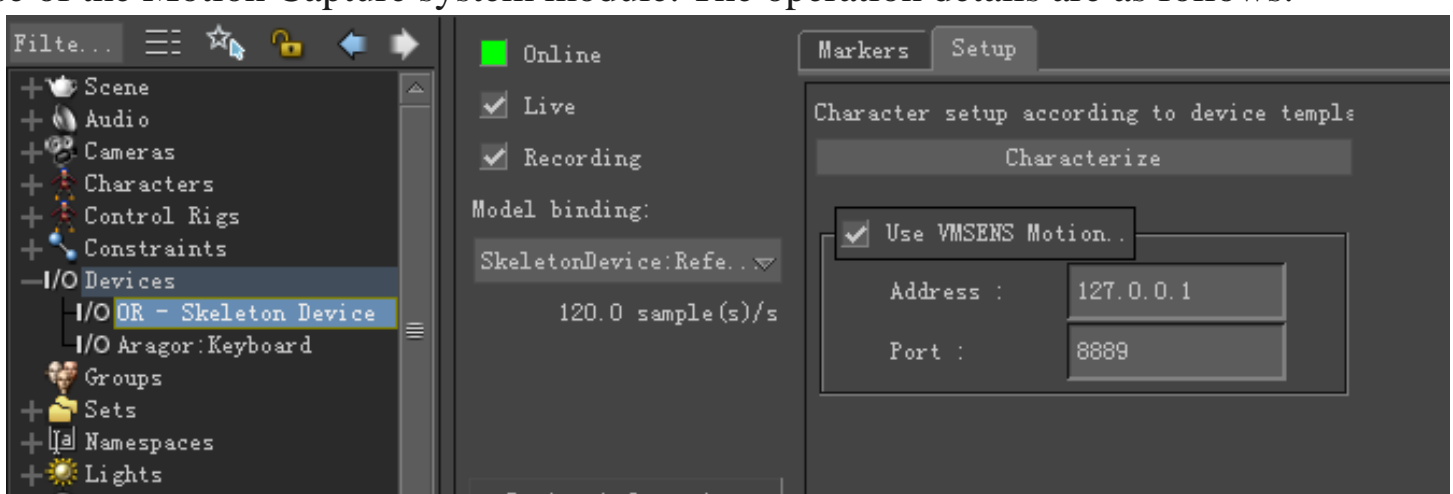

Figure 4 in the Motion Builder software socket communication set the network address and port number of the child window

\section{Conclusion}

Motion capture technology has been widely used in the animation industry and film and television industry, greatly improved the efficiency of production and save a huge cost. Human motion capture 
virtual interactive system, not only can be used for the animation industry, film and television industry, and even in the future of virtual reality application development, virtual teaching, virtual training system can also get more features and application development, saving users a lot of time and costs.

\section{Acknowledgements}

2016 Lanzhou Jiaotong University undergraduate teaching reform project "animation professional talent training program research and practice under animation industry background"

Project number: JGY201616

2014 Gansu natural science fund project "Human motion capture system design based on micro-mechanical sensor and Motion Builder"

Project number: 148RJZA032

\section{References}

[1] Huang Boshi, Chen Fumin. Research on Human Motion Capture and Motion Control. Computer Engineering and Applications, 2005, (07): 60-63

[2] Li Jia. Multi-perspective three-dimensional human motion capture research, Beijing Jiaotong University doctoral dissertation, 2013

[3] Li Shuai. Design of Human Motion Capture System Based on Micro-mechanical Sensor and Motion Builder. Master's Degree Paper, Lanzhou Jiaotong University, 2014 